\title{
Characterizations for New Classes of Analytic Functions Defined By Using Salagean Operator
}

\author{
Oyekan Ezekiel Abiodun ${ }^{1}$, Adelodun Olabode Paul ${ }^{2}$ and Ajai, Philip Terwase \\ ${ }^{1,2}$ (Department of Mathematics \& Statistics/Bowen University Iwo, Nigeria) \\ ${ }^{3}$ (Department of Mathematics /Plateau State University Bokkos, Nigeria)
}

Abstract : In this paper, we study certain subclasses $U_{m, n}(\beta, A, B, \rho)$ and $U_{m, n}^{*}(\beta, A, B, \rho)$ of analytic functions in the unit disk. The results presented include coefficient estimates and several subordination properties for functions belonging to these subclasses. Our results extend some earlier works.

Keywords: subordinating factor sequence, univalent, convex, analytic, convolution (or Hadamard product)

\section{Introduction}

Let $\mathbf{A}$ denote the class of functions of the form

$f(z)=z+\sum_{k=2}^{\infty} a_{k} z^{k}$

that are analytic and univalent in the open unit disk $U=\{z \in \mathbf{C} ;|z|<1\}$. Let $g(z) \in \mathbf{A}$ be given by

$$
g(z)=z+\sum_{k=2}^{\infty} b_{k} z^{k}
$$

Furthermore, let

$$
\begin{aligned}
& \Phi(z)=z+\sum_{k=2}^{\infty} \lambda_{k} z^{k}, \lambda \geq 0 \\
& \Phi(z)=z+\sum_{k=2}^{\infty} \mu_{k} z^{k}, \mu \geq 0
\end{aligned}
$$

Which are analytic and normalized by the conditions that $f(0)=f^{\prime}(0)-1=0$

For $f(z) \in \mathbf{A}$, Salagean [1] introduced the following differential operator,

$$
D^{0} f(z)=f(z), D^{\prime} f(z)=z f^{\prime}(z), \ldots, D^{n} f(z)=D\left(D^{n-1} f(z)\right)(n \in \mathbf{N}=\{1,2, \ldots\}) \text {. }
$$

We note that

$$
D^{n} f(z)=z+\sum_{k=2}^{\infty} k^{n} a_{k} z^{k}\left(n \in \mathbf{N}_{0}=\mathbf{N} \cup\{0\}\right)
$$

Definition 1 (Hardamard Product or Convolution)

Given two functions $f$ and $g$ in the class $\mathbf{A}$, where $f(z)$ and $g(z)$ are given by (1.1) and (1.2) respectively, the Hardamard product (or Convolution) of $f$ and $g$ is defined (as usual) by

$$
(f * g)(z)=z+\sum_{k=2}^{\infty} a_{k} b_{k} z^{k}=(g * f)(z)
$$

Definition 2 (Subordination Principle)

For two functions $f$ and $g$, analytic in $U$,we say that the function $f(z)$ is subordinate to $g(z)$ in $U$, and write $f(z) \prec g(z)$ if there exists a Schwarz function $\omega(z)$ which (by definition) is analytic in $U$ with $\omega(0)=0$ and $|\omega(z)|<1$ such that $f(z)=g(\omega(z))(z \in U)$. Indeed it is known that $f(z) \prec g(z) \Rightarrow f(0)=g(0)$ and $f(u) \subset(g(u))$

Furthermore, if the function $g$ is univalent in $U$, then we have the following equivalence [8, p. 4]: 
Definition 3 [3]

Let $U_{m, n}(\beta, A, B)$ denote the subclasses of A consisting of functions $f(z)$ of the form $(1.1)$ and satisfy the following subordination:

$$
\begin{aligned}
& \frac{D^{m} f(z)}{D^{n} f(z)}-B\left|\frac{D^{m} f(z)}{D^{m} f(z)}-1\right| \prec \frac{1+A z}{1+B z} \\
& \left(-1 \leq B<A \leq 1 ; \beta \geq 0 ; m \in \mathbf{N}_{0}, m>n ; z \in u\right)
\end{aligned}
$$

\section{Remark 1}

By specializing the parameters $A, B, \beta, m$ and $n$, certain subclasses studied by various authors are obtained. For instance,

(i) $U_{m, n}(\beta, 1-2 \alpha,-1)=N_{m, n}(\alpha, \beta)$ (see Eker and Owa [4])

(ii) $U_{m+1, n}(\beta, 1-2 \alpha,-1)=S(n, \alpha, \beta)$ (see Rosy and Mumgundaramorthy [5], Asurf [6])

(iii) $U_{1,0}(\beta, 1-2 \alpha,-1)=U S(\alpha, \beta)$ (see Shaw et al [7])

(iv) $U_{2,1}(\beta, 1-2 \alpha,-1)=U K(\alpha, \beta)$ (see Shaw and Kulkarni[8])

(v) $U_{1,0}(0, A, B)=S^{*}(A, B), U_{2,1}(0, A, B)=K(A, B)$ (see Jarowski [9] and Padmanashon and [10])

Therefore, in the view of (1.3), definitions 1 and 3, we now give the following definitions:

\section{Definition 4}

Let $U_{m, n}(\beta, A, B, \rho)$ denote the subclasses of A consisting of functions $f(z)$ of the form (1.1) and satisfying the following condition;

$$
\begin{aligned}
& \left|\frac{D^{m}(f * \Phi)^{\rho}(z)}{D^{n}\left(f^{*} \psi\right)^{\rho}(z)}\right|-B\left|\frac{D^{m}\left(f^{*} \Phi\right)^{\rho}(z)}{D^{n}\left(f^{*} \psi\right)^{\rho}(z)}-1\right| \prec \frac{1+A z}{1+B z} \\
& \left(-1 \leq B<A \leq 1 ; \beta \geq 0 ; m, \rho \in \mathbf{N} ; n \in \mathbf{N}_{0}=\mathbf{N} \cup\{0\}\right), m>n ; z \in U
\end{aligned}
$$

Also, we note that for $\lambda_{k} \geq \mu_{k} \geq 0$, when $\lambda_{k}=\mu_{k}=1$ then, $\Phi(z)=\psi(z)=\frac{z}{1-z} \in K$ such that our

Definition 4 will be equivalent to Definition 3. This is because

$$
(f * \Phi)(z)=(f * \psi)(z)=f
$$

Definition 5 (Subordinating Factor Sequence)

A sequence $\left\{c_{k}\right\}_{k=0}^{\infty}$ of complex numbers is said to be a subordinating factor sequence if, whenever $f(z)$ of the form (1.1) is analytic, univalent and convex in $U$, we have the subordination given by;

$$
\sum_{k=1}^{\infty} a_{k} c_{k} z^{k} \prec f(z) \quad\left(a_{1}=1, z \in U\right)
$$

\section{Main Results}

Unless otherwise stated, we shall in the sequence assume that

$-1 \leq B<A \leq 1, \beta \geq 0, m, \rho \in \mathbf{N}, n \in \mathbf{N}_{0}, m>n, a_{k}(\rho) \geq 0, \lambda_{k}(\rho) \geq 0, \mu_{k}(\rho) \geq 0$; where $a_{k}(\rho), \lambda_{k}(\rho), \mu_{k}(\rho)$ are coefficients of $a_{k}, \lambda_{k}, \mu_{k}$ all depending on $\rho$ and $z \in U$.

We now prove the following theorem which gives a sufficient condition for functions belonging to the class $U_{m, n}(\beta, A, B, \rho)$. 


\section{Theorem 1}

A function $f(z)$ of the form (1.1) is in the class $U_{m, n}(\beta, A, B, \rho)$

if; $\sum_{k=2}^{\infty}\left\{(1+\beta(1+|B|) \mid](\rho+k-1)^{m} \lambda_{k}(\rho)-(\rho+k-1)^{n} \mu_{k}(\rho)\right]+\left|B(\rho+k-1)^{m} \lambda_{k}(\rho)-A(\rho+k-1)^{n} \mu_{k}(\rho)\right|\left|a_{k}(\rho)\right| \leq\left(A \rho^{n}-B \rho^{m}\right)-\left[1+\beta(1+|B|) \mid\left(\rho^{n}-\rho^{m}\right)\right.$

\section{Proof}

It suffices to show that

$$
\left|\frac{P(z)-1}{A-B p(z)}\right|<1
$$

Where

$$
P(z)=\frac{D^{m}(f * \Phi)^{\rho}(z)}{D^{n}\left(f^{*} \psi\right)^{\rho}(z)}-B\left|\frac{D^{m}(f * \Phi)^{\rho}(z)}{D^{n}\left(f^{*} \psi\right)^{\rho}(z)}-1\right|
$$

We have

$$
\left|\frac{P(z)-1}{A-B p(z)}\right|=\left|\frac{D^{m}(f * \Phi)^{\rho}(z)-\beta e^{i \theta}\left|D^{m}(f * \Phi)^{\rho}(z)-D^{n}(f * \psi)^{\rho}(z)\right|-D^{n}(f * \psi)^{\rho}(z)}{A D^{m}\left(f^{*} \Phi\right)^{\rho}(z)-B\left[D^{m}(f * \Phi)^{\rho}(z)-\beta e^{i \theta}\left|D^{m}(f * \Phi)^{\rho}(z)-D^{n}(f * \psi)^{\rho}(z)\right|\right]}\right|
$$$$
=\left|\frac{\left(\rho^{n}-\rho^{m}\right) z^{\rho}+\sum_{k=2}^{\infty}\left[(\rho+k-1)^{m} \lambda_{k}(\rho)-(\rho+k-1)^{n} \mu_{k}(\rho)\right] a_{k}(\rho) z^{\rho+k-1}+\beta e^{i \theta} \mid\left(\rho^{n}-\rho^{m}\right) z^{\rho}+\sum_{k=2}^{\infty}\left[(\rho+k-1)^{m} \lambda_{k}(\rho)-(\rho+k-1)^{n} \mu_{k}(\rho) a_{k}(\rho) z^{\rho+k-1} \mid\right.}{\left(A \rho^{n}-B \rho^{m}\right)^{\rho}-\sum_{k=2}^{\infty}\left[B(\rho+k-1)^{m} \lambda_{k}(\rho)-A(\rho+k-1)^{n} \mu_{k}(\rho) a_{k}(\rho) z^{\rho+k-1}-B \beta e^{i \theta}\left|\left(\rho^{n}-\rho^{m}\right) z^{\rho}+\sum_{k=2}^{\infty}\left[(\rho+k-1)^{m} \lambda_{k}(\rho)-(\rho+k-1)^{n} \mu_{k}(\rho)\right]_{k}(\rho) z^{\rho+k-1}\right|\right.}\right|
$$

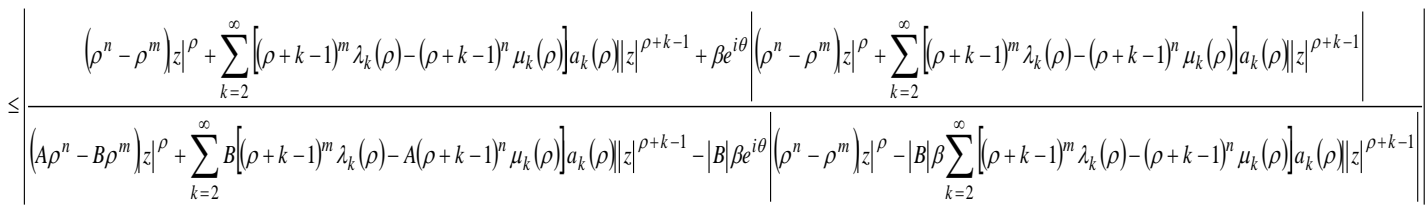

$$
\leq\left|\frac{\left(\rho^{n}-\rho^{m}\right)+\sum_{k=2}^{\infty}\left[(\rho+k-1)^{m} \lambda_{k}(\rho)-(\rho+k-1)^{n} \mu_{k}(\rho)\right] a_{k}(\rho)+\beta\left(\rho^{n}-\rho^{m}\right)+\sum_{k=2}^{\infty}\left[(\rho+k-1)^{m} \lambda_{k}(\rho)-(\rho+k-1)^{n} \mu_{k}(\rho)\right] a_{k}(\rho) \mid}{\left(A \rho^{n}-B \rho^{m}\right)|z|^{\rho}+\left.\sum_{k=2}^{\infty} B\left[(\rho+k-1)^{m} \lambda_{k}(\rho)-A(\rho+k-1)^{n} \mu_{k}(\rho)\right] a_{k}(\rho)|-| B|\beta|\left(\rho^{n}-\rho^{m}\right) z\right|^{\rho}-|B| \beta \sum_{k=2}^{\infty}\left[(\rho+k-1)^{m} \lambda_{k}(\rho)-(\rho+k-1)^{n} \mu_{k}(\rho)\right] a_{k}(\rho) \mid}\right| \mid
$$

This last expression is bounded above by 1 if

$$
\mid \frac{\left(\rho^{n}-\rho^{m}\right)+\sum_{k=2}^{\infty}\left[(\rho+k-1)^{m} \lambda_{k}(\rho)-(\rho+k-1)^{n} \mu_{k}(\rho)\right] a_{k}(\rho)+\beta\left(\rho^{n}-\rho^{m}\right)+\sum_{k=2}^{\infty}\left[(\rho+k-1)^{m} \lambda_{k}(\rho)-(\rho+k-1)^{n} \mu_{k}(\rho)\right] a_{k}(\rho) \mid}{\left|\left(A \rho^{n}-B \rho^{m}\right) z\right|^{\rho}+\sum_{k=2}^{\infty} B\left[(\rho+k-1)^{m} \lambda_{k}(\rho)-A(\rho+k-1)^{n} \mu_{k}(\rho)\right] a_{k}(\rho)-|B| \beta\left(\rho^{n}-\rho^{m}\right) z^{\rho}-|B| \beta \sum_{k=2}^{\infty}\left[(\rho+k-1)^{m} \lambda_{k}(\rho)-(\rho k-1)^{n} \mu_{k}(\rho)\right] a_{k}(\rho)||} \leq 1
$$

i.e. that

$$
\sum_{k=2}^{\infty}\left\{\left[1+\beta(1+|B|)\left|\left[(\rho+k-1)^{m} \lambda_{k}(\rho)-(\rho+k-1)^{n} \mu_{k}(\rho)\right]+\right| B(\rho+k-1)^{m} \lambda_{k}(\rho)-A(\rho+k-1)^{n} \mu_{k}(\rho) \mid\right)\left|a_{k}(\rho)\right| \leq\left(A \rho^{n}-B \rho^{m}\right)-\left[1+\beta(1+|B|) \mid\left(\rho^{n}-\rho^{m}\right)\right.\right.
$$

and hence, the proof of Theorem 1 is obtained.

By taking $\rho=1$ in theorem 1 when $\lambda_{k}(\rho)=\mu_{k}(\rho)=1$ and $a_{k}(\rho)$ is the coefficient $a_{k}$ depending on $\rho$, we obtain the following

\section{Corollary 1}

A function $f(z)$ of the form (1.1) is in the class of $U_{m, n}(\beta, A, B, \rho)$ if

$$
\sum_{k=2}^{\infty}\left[(1+(1+|B|))\left(k^{m}-k^{n}\right)+\left|B k^{m}-A k^{n}\right|\right] a_{k} \mid \leq A-B
$$


This means that

$$
U_{m, n}(\beta, A, B, 1)=U_{m, n}(\beta, A, B)=\left\{f \in A: \frac{D^{m} f(z)}{D^{n} f(z)}-B\left|\frac{D^{m} f(z)}{D^{n} f(z)}-1\right| \prec \frac{1+A z}{1+B z}\right\}
$$

\section{Remark 2}

(i) The result in corollary 1 which is the correct result obtained by Li and Tang [3, theorem 1], is due to M.K. Aouf et al [11]

(ii)Putting $A=1-2 \alpha,(0 \leq \alpha<1), B=-1, m=n+1\left(n \in \mathbf{N}_{0}\right)$ and $\rho=1$ we obtain the result due to Rosy and Murugusudaramworthy [3, theorem 2]

Let $U_{m, n}^{*}(\beta, A, B, \rho)$ denote the class of $f(z \in \mathbf{A})$ whose coefficients satisfy the condition (2.1).

We note that $U_{m, n}^{*}(\beta, A, B, \rho) \subseteq U_{m, n}(\beta, A, B, \rho)$.

By employing the technique used earlier by [12] and Srivastava [13], we now state and prove our next result; which is a subordination result for the class $U_{m, n}^{*}(\beta, A, B, \rho)$. However, we first give the following lemma which is required for the proof of our next theorem.

\section{Lemma 1 [14]}

The sequence $\left\{c_{k}\right\}_{k=0}^{\infty}$ is a subordinating factor sequence if and only if

$$
\operatorname{Re}\left\{1+2 \sum_{k=1}^{\infty} c_{k} z^{k}\right\}>0 \quad(z \in U)
$$

\section{Theorem 2}

Let $f(z) \in U_{m, n}^{*}(\beta, A, B, \rho)$. Then

$$
2\left\{\frac{\Omega(2)}{\left.2\left(A \rho^{n}-B \rho^{m}\right)-[1+\beta(1+|B|))\left(\rho^{m}-\rho^{n}\right)+\Omega(2)\right\}}\left(f^{*} h\right) \prec h(z) \quad(z \in U)\right.
$$

for every function $h \in k$ and

$$
\operatorname{Re}\{f(z)\}>-\frac{\left.\left(A \rho^{n}-B \rho^{m}\right)-[1+\beta(1+|B|))\right]\left(\rho^{m}-\rho^{n}\right)+\Omega(2)}{\Omega(2)}
$$

The constant factor $\frac{\Omega(2)}{2\left(\left(A \rho^{n}-B \rho^{m}\right)-[1+\beta(1+|B|)]\left(\rho^{m}-\rho^{n}\right)+\Omega(2)\right\}}$ in subordination result (2.3) cannot be replaced by a larger one.

\section{Proof}

Let $f(z) \in U_{m, n}^{*}(\beta, A, B, \rho)$ and let $h(z)=z+\sum_{k=2}^{\infty} c_{k} z^{k} \in$. Then we have

$$
2\left\{\frac{\Omega(2)}{\left.2\left(A \rho^{n}-B \rho^{m}\right)-[1+\beta(1+|B|)]\left(\rho^{m}-\rho^{n}\right)+\Omega(2)\right\}}\left(f^{*} h\right)(z)\right.
$$

Thus by definition 5 , the subordination result (2.3) will hold if the sequence

$$
\left.\left\{\frac{\Omega(2)}{2\left\{\left(A \rho^{n}-B \rho^{m}\right)-[1+\beta(1+|\beta|)]\left(\rho^{m}-\rho^{n}\right)+\Omega(2)\right\}}\right\}\right\}_{k=1}^{\infty}
$$

Is a subordinating factor sequence with $a_{1}=1$. In the view of lemma 1 , this is equivalent to the following inequality; 
$\operatorname{Re}\left\{1+\sum_{k=1}^{\infty} \frac{\Omega(2)}{\left(A \rho^{n}-B \rho^{m}\right)-\left[1+\beta\left(1+|B| \mid\left(\rho^{m}-\rho^{n}\right)+\Omega(2)\right.\right.} a_{k} z^{k}\right\}>0(z \in \mathbf{U})$

Now, since

$\Omega(k)=\left\{\left[1+\beta(1+|B|)\left|\left[(\rho+k-1)^{m} \lambda_{k}(\rho)-(\rho+k-1)^{n} \mu_{k}(\rho)\right]+\right| B(\rho+k-1)^{m} \lambda_{k}(\rho)-A(\rho+k-1)^{n} \mu_{k}(\rho) \mid\right\}\right.$

is an increasing function of $k,(k \geq 2)$, we have

$\operatorname{Re}\left\{1+\sum_{k=1}^{\infty} \frac{\Omega(2)}{\left.\left(\rho^{n}-B \rho^{m}\right)-[1+\beta(1+\mid B]] \rho^{m}-\rho^{n}\right)+\Omega(2)} a_{k} z^{k}\right\}=\operatorname{Re}\left\{1+\frac{\Omega(2)}{\left(A \rho^{n}-B \rho^{m}\right)-\left[1+\beta(1+\mid B B] \rho^{m}-\rho^{n}\right)+\Omega(2)} z+\frac{1}{\left.\left(A \rho^{n}-B \rho^{m}\right)-[1+\beta(1+\mid B]] \rho^{m}-\rho^{n}\right)+\Omega(2)} \sum_{k=2}^{\infty} \Omega(2) a_{k} z^{k}\right\}$

$\geq 1-\frac{\Omega(2)}{\left(A \rho^{n}-B \rho^{m}\right)-\left[1+\beta\left(1+|B| \mid\left(\rho^{m}-\rho^{n}\right)+\Omega(2)\right.\right.} r-\frac{1}{\left(A \rho^{n}-B \rho^{m}\right)-\left[1+\beta\left(1+|B| \mid\left(\rho^{m}-\rho^{n}\right)+\Omega(2)\right.\right.} \sum_{k=2}^{\infty} \Omega(k)\left|a_{k}\right| r^{k}$

$>1-\frac{\Omega(2)}{\left(A \rho^{n}-B \rho^{m}\right)-\left[1+\beta\left(1+|B|\left(\rho^{m}-\rho^{n}\right)+\Omega(2)\right.\right.} r-\frac{\left(A \rho^{n}-B \rho^{m}\right)-\left[1+\beta(1+|B|]\left(\rho^{m}-\rho^{n}\right)\right.}{\left(A \rho^{n}-B \rho^{m}\right)-\left[1+\beta(1+|B|]\left(\rho^{m}-\rho^{n}\right)+\Omega(2)\right.} r$

$=1-r>0 \quad(|z|=r<1)$

where we have also made use of the assertion (2.1) of theorem 1. Thus (2.6) holds this proves the inequality

(2.3). The inequality (2.4) follows from (2.3) by taking the convex function

$h(z)=\frac{z}{1-z}=z+\sum_{k=2}^{\infty} z^{k}$

To prove the constant

$\frac{\Omega(2)}{2\left\{\left(A \rho^{n}-B \rho^{m}\right)-\left[1+\beta(1+|B|]\left(\rho^{m}-\rho^{n}\right)+\Omega(2)\right\}\right.}$

We consider the function

$f_{0}(z) \in U_{m, n}^{*}(\beta, A, B, \rho)$ given by

$f_{0}(z)=z-\frac{\left(A \rho^{n}-B \rho^{m}\right)-\left[1+\beta(1+|B|]\left(\rho^{m}-\rho^{n}\right)\right.}{\left\{\left[1+\beta(1+|B|] \mid\left[(\rho+1)^{n} \lambda_{2}(\rho)-(\rho+1)^{n} \mu_{2}(\rho)+\right]+\left[B(\rho+1)^{m} \lambda_{2}(\rho)-A(\rho+1)^{n} \mu_{2}(\rho)\right]\right\}^{z^{2}}\right.}$

Thus from (2.3)

$\frac{\Omega(2)}{2\left\{\left(A \rho^{n}-B \rho^{m}\right)-\left[1+\beta\left(1+|B| \mid\left(\rho^{m}-\rho^{n}\right)+\Omega(2)\right\}\right.\right.} f_{0}(z) \prec \frac{z}{1-z} \quad(z \in \mathbf{U})$

Moreover, it can easily be verified for functions $f_{0}(z)$ given by (2.7) that

$\min _{|z| \leq r}\left\{\operatorname{Re} \frac{\Omega(2)}{2\left(\left(A \rho^{n}-B \rho^{m}\right)-\left[1+\beta(1+|B|]\left(\rho^{m}-\rho^{n}\right)+\Omega(2)\right\}\right.} f_{0}(z)\right\}=-\frac{1}{2}$

This show that the constant

$\frac{\Omega(2)}{2\left\{\left(A \rho^{n}-B \rho^{m}\right)-\left[1+\beta(1+|B|]\left(\rho^{m}-\rho^{n}\right)+\Omega(2)\right\}\right.}$ is the best possible.

This completes the proof of theorem 2 .

Remark 3: $\quad$ When $\lambda_{2}(\rho)=\mu_{2}(\rho)=1 \quad\left(\lambda_{k}(\rho) \geq \mu_{k}(\rho) \geq 0\right)$

(i)Taking $A=1-2 \alpha \quad(0 \leq \alpha<1), B=-1$ and $\rho=1$ in theorem 2, we correct the result obtained by Srivastava and Eker [15, Theorem 1]

(ii) Taking $A=1-2 \alpha \quad(0 \leq \alpha<1), B=-1, m=n+1$ and $\rho=1$ in theorem 3, we obtain the result obtained by Aouf et al. [16, Corollary 4] 
(iii) Taking $A=1-2 \alpha \quad(0 \leq \alpha<1), B=-1, m=1, n=0$ and $\rho=1$ in theorem 2, we obtain the result obtained by Frasin [17, Corollary 2.2]

(iv) Taking $A=1-2 \alpha \quad(0 \leq \alpha<1), B=-1, m=2$, and $n=\rho=1$ in theorem 3 we obtain the result obtained by Frasin [17, Corollary 2.5]

(v) Taking $\beta=\alpha \geq 0, B=-1, \rho=1$ and $\lambda_{2}(\rho)=\mu_{2}(\rho)=1$ in theorem 3, we obtain the result obtained by Oyekan and Opoola [18, Theorem 2.1]

\section{References}

[1]. G.S. Salagean, Subclasses of Univalent Functions, Lecture Notes in Math (Springer-verlag), (1983) 362-372

[2]. S.S Miller and P.T Mocanu, Differential Subordinations: Theory and Applications, Series on Monographs and textbooks in pure and applied maths, No. 225 Marcel Dekker Inc., New York 2000.

[3]. S.H Li and H. Tang, Certain new Classes of Analytic functions defined by using Salagean operator, Bull. Math. Anal. Appl., 2 (4), 2010, 62-75

[4]. S.S Eker and S. Owa, Certain classes of analytic functions involving Salagean operator, J Inequal. Pure \& Appl Math. 10(1), 2009, $12-22$

[5]. T. Rosy and G. Murugusundaramoorthy, Fractional calculus and their applications to certain subclasses of uniformly convex functions, Far East J. math sci., 15 (2), 2004, 231-242

[6]. M.K Aouf, A subclass of uniformly convex functions with negative coefficients, Math (cluj), 52(3), 2010, 99-11

[7]. S. Shams, S.R Kulkarni and J. M Jahangiri, Classes of uniformly starlike and functions, Internat. J. math Math sci., 55, 2004, 29592961

[8]. S. Shams and S.R Kulkarni, On a class of univalent functions defined by Ruscheweyh derivatives, Kyungpook Math J. 43, 2009, 579-585

[9]. W. Janowski, Some extreme problem for certain families of analytic functions, Ann. Polon. Math. 28, 1973, 648-658

[10]. K.S. Padmanabhan and M.S Ganesan, Convolution of certain classes of univalent function with negative coefficients, Indian J. Pure Appl math., 19(9), 1988, 880-889

[11]. M.K Aouf, R.M El-Ashwah, A.A.M Hassan and A.H Hassan, On subordination results for certain new classes of analytic functions defined by using Salagean operator, Bull. Of Math. Anal. and Appl., 4(1), 2012, 239-246.

[12]. A.A Attiya, On some application of subordination theorems, J math Anal. Appl 311, 2005, 489-494

[13]. H.M Srivastava and A.A Attiya, Some subordination results associated with certain subclasses of analytic functions, J. Inequal Pure Math Sci., 5 4, 2004, 1-6

[14]. H.S Wilf, Subordinating factor sequence for convex maps of unit circle, Proc. Amer. Math. Soc. 12, 1961, 689-693

[15]. H.M Srivastava and S.S Eker, Some applications of a subordination theorem for a class of analytic functions, Appl. Math. Letters 21, 2008, 394-399

[16]. M.K Aouf, R.M El-Ashwah and S.M el-Deeb, Subordination results for some subclasses of uniformly starlike and convex function defined by convolution, European J Pure Appl Math 3(5), 2010, 903-917

[17]. B.A Frasin, Subordination results for a class of analytic functions defined by a linear operator, J. Inequal pure Appl math. 7(4), 2006, 1-7

[18]. E.A Oyekan and T.O Opoola, Some subordination results for certain analytic functions defined by using salagean operator, Amer. J. Mathematics and Statistics, 4(6), 2013 